ANNALES

POLONICI MATHEMATICI

$81.1(2003)$

\title{
Einstein-Hermitian and anti-Hermitian 4-manifolds
}

\author{
by WŁodZimierz Jelonek (Kraków)
}

\begin{abstract}
We study 4-dimensional Einstein-Hermitian non-Kähler manifolds admitting a certain anti-Hermitian structure. We also describe Einstein 4-manifolds which are of cohomogeneity 1 with respect to an at least 4-dimensional group of isometries.
\end{abstract}

0. Introduction. Einstein-Hermitian non-Kähler surfaces are recently a subject of intensive investigation (see $[\mathrm{LeB}],[\mathrm{G}-\mathrm{M}],[\mathrm{C}-\mathrm{S}-\mathrm{V}],[\mathrm{P}-\mathrm{P}]$, [A-G-2]). LeBrun has proved that every such compact surface is a blow-up of $\mathbb{C} P^{2}$ in at least three points and has necessarily a positive scalar curvature. He also showed that the only Einstein-Hermitian metric on the blow-up of $\mathbb{C} P^{2}$ at one point is D. Page's metric. Earlier Grantcharov and Muskarov $[\mathrm{G}-\mathrm{M}]$ investigated compact Hermitian surfaces which are $*$-Einstein. They proved that every such non-Kähler surface is conformal to an extremal Kähler metric with non-constant positive scalar curvature and has positive (clearly constant) scalar curvature. After the works of LeBrun [LeB], Apostolov and Gauduchon [A-G-1] and Cho, Sekigawa and Vanhecke [C-S-V] it is clear that every Einstein-Hermitian surface must be $*$-Einstein. Plebański and Przanowski [P-P] have given a local classification of Einstein-Hermitian surfaces which admit a Killing vector field.

With every Hermitian non-Kähler 4-manifold $(M, g, J)$ there are related two natural distributions $\mathcal{D}=\left\{X \in T M: \nabla_{X} J=0\right\}, \mathcal{D}^{\perp}=\{Y \in T M$ : $g(Y, X)=0$ for all $X \in \mathcal{D}\}$ defined in the open set $U=\left\{x:\left|\nabla J_{x}\right| \neq 0\right\}$. These distributions are $J$-invariant and on $U$ we can define the opposite almost Hermitian structure $\bar{J}$ by $\bar{J} X=J X$ if $X \in \mathcal{D}^{\perp}$ and $\bar{J} X=-J X$ if $X \in \mathcal{D}$; we call it the natural opposite almost Hermitian structure. It is not difficult to check that for the famous Einstein-Hermitian manifold $\mathbb{C} P^{2} \sharp \overline{\mathbb{C} P^{2}}$ with D. Page's metric (see $[\mathrm{P}],[\mathrm{B}],[\mathrm{K}]$ ) the opposite structure $\bar{J}$ is Hermitian and this structure extends to a global opposite Hermitian structure.

2000 Mathematics Subject Classification: 53B21, 53B35, 53C25.

Key words and phrases: Einstein 4-manifolds, Hermitian surface, cohomogeneity 1 manifold, $J$-invariant Ricci tensor. 
Natural questions arise for general Einstein-Hermitian non-Kähler manifolds: When is $\bar{J}$ Hermitian? Under what conditions does it extend to a global opposite Hermitian structure? The first question in the case of self-dual Einstein-Hermitian 4-manifolds was recently answered by Apostolov and Gauduchon [A-G-2]. We give the answers to these questions for compact Einstein-Hermitian manifolds and partial answers for arbitrary Einstein-Hermitian surfaces. Our method is based on introducing a special orthonormal frame naturally related to the Hermitian structure $J$ and the metric $g$. We show that for Einstein 4-manifolds the set $U$ is dense and the set $\left\{x:\left|\nabla J_{x}\right|=0\right\}$ is a totally geodesic submanifold of $(M, g)$. We prove that the opposite almost Hermitian structure $\bar{J}$ of an Einstein-Hermitian non-ASD surface is Hermitian if and only if the metric $g$ is of cohomogeneity 1 . We also give a local description of non-Kähler non-locally symmetric Einstein-Hermitian surfaces admitting an opposite Hermitian structure as products $\mathbb{R} \times P_{0}$ where $P_{0}$ is a 3-dimensional naturally reductive manifold, and prove that they are always of cohomogeneity 1 with the group of local isometries of dimension at least 4 . We show that if $(M, g, J)$ is a compact Einstein-Hermitian non-Kähler manifold for which $\bar{J}$ is integrable then $\bar{J}$ extends to a global structure and $(M, g, J)$ is isometrically biholomorphic to $\mathbb{C} P^{2} \sharp \overline{\mathbb{C} P^{2}}$ with D. Page's metric. From the result of LeBrun it easily follows that every Hermitian non-Kähler Einstein manifold which admits an opposite Hermitian structure must be biholomorphic to $\mathbb{C} P^{2} \sharp \overline{\mathbb{C} P^{2}}$ with D. Page's metric so the above result for compact surfaces is a simple consequence of $[\mathrm{LeB}]$.

1. Hermitian 4-manifolds. Let $(M, g, J)$ be an almost Hermitian manifold, i.e. $J$ is an almost complex structure orthogonal with respect to $g$, i.e. $g(X, Y)=g(J X, J Y)$ for all $X, Y \in \mathfrak{X}(M)$. We say that $(M, g, J)$ is a Hermitian manifold if its almost Hermitian structure $J$ is integrable. Set $\bigwedge^{2} M=\bigwedge^{2} T^{*} M$. In what follows we identify the bundle $T M$ with $T^{*} M$ by means of $g$, so we also write $\bigwedge^{2} M=\bigwedge^{2} T M$. The Hodge star operator $*$ (which depends on the orientation of $M$ ) defines an endomorphism $*: \bigwedge^{2} M \rightarrow \bigwedge^{2} M$ with $*^{2}=$ id and we denote by $\bigwedge^{+}, \bigwedge^{-}$its eigensubbundles corresponding to $1,-1$ respectively.

In what follows we consider 4-dimensional Hermitian manifolds $(M, g, J)$ which we also call Hermitian surfaces. Such manifolds are always oriented and we choose an orientation in such a way that the Kähler form $\Omega(X, Y)=$ $g(J X, Y)$ is self-dual (i.e. $\left.\Omega \in \wedge^{+} M\right)$. The vector bundle of self-dual forms admits a decomposition

$$
\bigwedge^{+} M=\mathbb{R} \Omega \oplus L M
$$

where $L M$ denotes the bundle of real $J$-skew invariant 2 -forms (i.e. $L M=$ 
$\{\Phi \in \bigwedge M: \Phi(J X, J Y)=-\Phi(X, Y)\})$. The bundle $L M$ is a complex line bundle over $M$ with the complex structure $\mathcal{J}$ defined by $(\mathcal{J} \Phi)(X, Y)=$ $-\Phi(J X, Y)$. For a 4-dimensional Hermitian manifold the covariant derivative of the Kähler form $\Omega$ is locally expressed by

$$
\nabla \Omega=a \otimes \Phi+\mathcal{J} a \otimes \mathcal{J} \Phi,
$$

where $\mathcal{J} a(X)=-a(J X)$. The Lee form $\theta$ of $(M, g, J)$ is defined by the equality $d \Omega=\theta \wedge \Omega$. We have $\theta=-\delta \Omega \circ J$. By $\varrho$ we denote the Ricci tensor of a Riemannian manifold $(M, g)$ and by $\tau$ the scalar curvature of $(M, g)$, i.e. $\tau=\operatorname{tr}_{g} \varrho$. A Hermitian manifold $(M, g, J)$ is said to have Hermitian Ricci tensor if $\varrho(X, Y)=\varrho(J X, J Y)$ for all $X, Y \in \mathfrak{X}(M)$. An involutive distribution is called a foliation. A foliation $D$ is called minimal if each of its leaves is a minimal submanifold of $(M, g)$, i.e. the trace of its second fundamental form (the mean curvature) vanishes. A Hermitian 4-manifold $(M, g, J)$ is said to have an opposite Hermitian structure if it admits an orthogonal Hermitian structure $\bar{J}$ with anti-self-dual Kähler form $\bar{\Omega}$. We then call $(M, g, J)$ an anti-Hermitian manifold with anti-Hermitian structure $\bar{J}$. For any almost Hermitian 4-manifold the following formula holds (see $[\mathrm{G}-\mathrm{H}])$ :

$$
\begin{aligned}
\frac{1}{2}(\varrho(X, Y)+\varrho(J X, J Y))-\frac{1}{2}\left(\varrho^{*}(X, Y)+\varrho^{*}(Y, X)\right) \\
=\frac{1}{4}\left(\tau-\tau^{*}\right) g(X, Y),
\end{aligned}
$$

where $\varrho^{*}$ is the $*$-Ricci tensor defined by

$$
\varrho^{*}(X, Y)=\frac{1}{2} \operatorname{tr}\{Z \mapsto R(X, J Y) J Z\},
$$

where $R(X, Y) Z=\left(\left[\nabla_{X}, \nabla_{Y}\right]-\nabla_{[X, Y]}\right) Z$ and $\tau^{*}=\operatorname{tr}_{g} \varrho^{*}$. By $\mathcal{D}$ we denote the nullity distribution of $(M, g, J)$ defined by $\mathcal{D}=\left\{X \in T M: \nabla_{X} J=0\right\}$. For a Hermitian manifold it follows from (1.2) that $\mathcal{D}$ is $J$-invariant. Consequently, $\operatorname{dim} \mathcal{D}=2$ in $M_{0}=\left\{x \in M: \nabla J_{x} \neq 0\right\}$. We call the nullity distribution involutive if $\mathcal{D}_{\mid M_{0}}$ is involutive. We denote by $\mathcal{D}^{\perp}$ the orthogonal complement of $\mathcal{D}$ in $M_{0}$.

The curvature tensor $R$ of a 4-dimensional manifold $(M, g)$ determines an endomorphism $\mathcal{R}$ of the bundle $\wedge^{2} M$ defined by $g(\mathcal{R}(X \wedge Y), Z \wedge W)=$ $\mathcal{R}(X \wedge Y, Z \wedge W)=-R(X, Y, Z, W)=-g(R(X, Y) Z, W)$. Note that $\varrho^{*}=$ $\mathcal{J R}(\Omega)$ and $\tau^{*}=2 \mathcal{R}(\Omega, \Omega)$. Set $\mathcal{R}_{\Lambda^{+}{ }_{M}}=p_{\Lambda^{+}{ }_{M}} \circ \mathcal{R}_{\mid \Lambda^{+}{ }_{M}}$ where $p_{\Lambda^{+}{ }_{M}}$ : $\bigwedge M \rightarrow \bigwedge^{+} M$ is the orthogonal projection. Then $\operatorname{tr} \mathcal{R}_{\Lambda^{+}{ }_{M}}=\tau / 4$. We also have (see $[\mathrm{C}-\mathrm{S}-\mathrm{V}, \mathrm{p} .16]$ )

$$
\frac{\tau-\tau^{*}}{2}=\delta \theta+2 \alpha^{2}
$$

where $\alpha^{2}=|\nabla J|^{2} / 8$. The conformal scalar curvature $\kappa$ is defined by (see 
[A-G-1, p. 425])

$$
\kappa=\tau-\frac{3}{2}\left(|\theta|^{2}+2 \delta \theta\right)=\frac{1}{2}\left(3 \tau^{*}-\tau\right) .
$$

We say that an almost Hermitian manifold $(M, g, J)$ satisfies the second condition $\left(G_{2}\right)$ of $A$. Gray if its curvature tensor $R$ satisfies

$\left(G_{2}\right) \quad R(X, Y, Z, W)-R(J X, J Y, Z, W)$

$$
=R(J X, Y, J Z, W)+R(J X, Y, Z, J W)
$$

for all $X, Y, Z, W \in \mathfrak{X}(M)$. We say that it satisfies the condition $\left(G_{3}\right)$ of A. Gray if

$$
R(J X, J Y, J Z, J W)=R(X, Y, Z, W)
$$

for all $X, Y, Z, W \in \mathfrak{X}(M)$. Define $B=\frac{1}{2}(\mathcal{R}-* \mathcal{R} *) ; W=\frac{1}{2}(\mathcal{R}+* \mathcal{R} *)_{0}=$ $\frac{1}{2}(\mathcal{R}+* \mathcal{R} *)-\frac{\tau}{12} \mathrm{Id} ; W^{+}=\frac{1}{2}(W+* W) ; W^{-}=\frac{1}{2}(W-* W)$. Then

$$
\mathcal{R}=\frac{\tau}{12} \mathrm{Id}+B+W^{+}+W^{-} .
$$

The tensor $W$ is called the Weyl tensor and its components $W^{+}, W^{-}$are called the self-dual and anti-self-dual Weyl tensors.

In what follows we use the following result of A. Derdziński (see [S-V, p. 219, Prop. 5] or [D-V, p. 476, Cor. 7.2]).

Proposition 1. Let $(M, g)$ be a 4-dimensional Einstein manifold such that $W \in \operatorname{End}\left(\bigwedge^{2} M\right)$ has constant eigenvalues. Then $(M, g)$ is locally symmetric.

2. Hermitian surfaces with Hermitian Ricci tensor. Note that for every manifold satisfying condition $\left(G_{3}\right)$ we have $\mathcal{R}(L M) \subset \bigwedge^{+} M$, its Ricci tensor $\varrho$ is $J$-invariant and its $*$-Ricci tensor is symmetric. Indeed, since $R(j(X \wedge Y), j(Z \wedge W))=R(X \wedge Y, Z \wedge W)$ where $j(X \wedge Y)=J X \wedge J Y$, we have $\mathcal{R}(\operatorname{ker}(j-\mathrm{id}), \operatorname{ker}(j+\mathrm{id}))=0$. Since $\operatorname{ker}(j-\mathrm{id})=\bigwedge^{-} M \oplus \mathbb{R} \Omega$ and $\operatorname{ker}(j+\mathrm{id})=L M$ we get $g\left(\mathcal{R}(L M), \bigwedge^{-} M \oplus \mathbb{R} \Omega\right)=0$. Consequently, $\mathcal{R}(L M) \subset L M \subset \bigwedge^{+} M$. In fact the condition $\mathcal{R}(L M) \subset \bigwedge^{+} M$ holds if and only if the Ricci tensor $\varrho$ of $(M, g)$ is $J$-invariant (see [D, p. 5, (i)]) and an almost Hermitian 4-manifold $(M, g, J)$ with $J$-invariant Ricci tensor and symmetric $*$-Ricci tensor satisfies $\left(G_{3}\right)$.

Lemma A. Let $(M, g, J)$ be a Hermitian 4-manifold. Assume that $|\nabla J|$ $\neq 0$ on $M$. Then for any local orthonormal oriented basis $\left\{E_{1}, E_{2}\right\}$ of $\mathcal{D}^{\perp}$ there exists a global oriented orthonormal basis $\left\{E_{3}, E_{4}\right\}$ of $\mathcal{D}$ independent of the choice of $\left\{E_{1}, E_{2}\right\}$ such that

$$
\nabla \Omega=\alpha\left(\theta_{1} \otimes \Phi+\theta_{2} \otimes \Psi\right),
$$


where $\Phi=\theta_{1} \wedge \theta_{3}-\theta_{2} \wedge \theta_{4}, \Psi=\theta_{1} \wedge \theta_{4}+\theta_{2} \wedge \theta_{3}, \alpha=\frac{1}{2 \sqrt{2}}|\nabla J|$ and $\left\{\theta_{1}, \theta_{2}, \theta_{3}, \theta_{4}\right\}$ is a cobasis dual to $\left\{E_{1}, E_{2}, E_{3}, E_{4}\right\}$. What is more, $\delta \Omega=$ $-2 \alpha \theta_{3}, \theta=-2 \alpha \theta_{4}$.

Proof. Let $\left\{E_{1}, E_{2}\right\}$ be any orthonormal basis of $\mathcal{D}^{\perp}, E_{2}=J E_{1}$. Then (1.2) holds where $a=\alpha \theta_{1}$. Choose any orthonormal basis $\left\{E_{3}^{\prime}, E_{4}^{\prime}=J E_{3}^{\prime}\right\}$ in $\mathcal{D}$. Define $\Phi^{\prime}=\theta_{1} \wedge \theta_{3}^{\prime}-\theta_{2} \wedge \theta_{4}^{\prime}, \Psi^{\prime}=\theta_{1} \wedge \theta_{4}^{\prime}+\theta_{2} \wedge \theta_{3}^{\prime}$. Then $\left\{\Phi^{\prime}, \Psi^{\prime}\right\}$ is an oriented orthonormal local basis in $L M$. Thus we have

$$
\Phi=(\cos \phi) \Phi^{\prime}-(\sin \phi) \Psi^{\prime}, \quad \Psi=(\sin \phi) \Phi^{\prime}+(\cos \phi) \Psi^{\prime}
$$

for some local function $\phi$. Then

$$
\nabla \Omega=\alpha\left\{\theta_{1}\left((\cos \phi) \Phi^{\prime}-(\sin \phi) \Psi^{\prime}\right)+\theta_{2}\left((\sin \phi) \Phi^{\prime}+(\cos \phi) \Psi^{\prime}\right)\right\} .
$$

Define $E_{3}=(\cos \phi) E_{3}^{\prime}-(\sin \phi) E_{4}^{\prime}, E_{4}=(\sin \phi) E_{3}^{\prime}+(\cos \phi) E_{4}^{\prime}$. Then $\left\{E_{3}, E_{4}\right\}$ is the basis we are looking for. From (2.1) it is easy to get $\delta \Omega=$ $-2 \alpha \theta_{3}, \theta=-2 \alpha \theta_{4}$.

Any frame $\left\{E_{1}, E_{2}, E_{3}, E_{4}\right\}$ constructed as above will be called standard (or special).

The following lemma is well known (it means that for a Hermitian surface the component $W_{3}^{+}$of the positive Weyl tensor vanishes).

Lemma B. Let $(M, g, J)$ be a Hermitian surface. Then for any local orthonormal basis $\{\Phi, \Psi\}$ of $L M$ we have $\mathcal{R}(\Phi, \Phi)=\mathcal{R}(\Psi, \Psi)$ and $\mathcal{R}(\Phi, \Psi)=0$.

It is known that a Hermitian manifold $(M, g, J)$ satisfies the second condition of Gray if and only if its Ricci tensor is $J$-invariant, it has symmetric *-Ricci tensor and the component $W_{3}^{+}$of the positive Weyl tensor vanishes (i.e. $\mathcal{R}_{L M}=a \operatorname{id}_{L M}$ where $\mathcal{R}_{L M}=p_{L M} \circ \mathcal{R}_{\mid L M}$ and $p_{L M}$ is the orthogonal projection $\left.p_{L M}: \bigwedge M \rightarrow L M\right)$. It is well known that any almost Hermitian manifold satisfying $\left(G_{2}\right)$ satisfies $\left(G_{3}\right)$ and that any Hermitian manifold satisfying $\left(G_{3}\right)$ satisfies $\left(G_{2}\right)$ (i.e. for Hermitian manifolds these two conditions are equivalent).

Lemma C. Let $(M, g, J)$ be a Hermitian surface with $J$-invariant Ricci tensor (i.e. $\left.\mathcal{R}(L M) \subset \Lambda^{+} M\right)$. Let $\left\{E_{1}, E_{2}, E_{3}, E_{4}\right\}$ be a local orthonormal frame such that (2.1) holds. Then

$$
\begin{gathered}
\Gamma_{11}^{3}=\Gamma_{22}^{3}=E_{3} \ln \alpha, \\
\Gamma_{44}^{3}=\Gamma_{21}^{4}=-\Gamma_{12}^{4}=-E_{3} \ln \alpha, \\
\Gamma_{21}^{3}=-\Gamma_{12}^{3}, \quad \Gamma_{11}^{4}=\Gamma_{22}^{4}, \\
-\Gamma_{21}^{3}+\Gamma_{22}^{4}=\alpha, \\
\Gamma_{33}^{4}=-E_{4} \ln \alpha+\alpha,
\end{gathered}
$$

where $\nabla_{X} E_{i}=\sum \omega_{i}^{j}(X) E_{j}$ and $\Gamma_{k j}^{i}=\omega_{j}^{i}\left(E_{k}\right)$. 
Proof. Note that $\Gamma_{k j}^{i}=-\Gamma_{k i}^{j}$. We have

$$
\begin{gathered}
g\left(\nabla_{E_{1}} J X, Y\right)=\alpha \Phi(X, Y), \quad g\left(\nabla_{E_{2}} J X, Y\right)=-\alpha \Psi(X, Y), \\
\nabla_{E_{3}} J=0, \quad \nabla_{E_{4}} J=0 .
\end{gathered}
$$

Write $p(X)=\frac{1}{2} g\left(\nabla_{X} \Phi, \Psi\right)=\omega_{1}^{2}(X)+\omega_{3}^{4}(X)$. Then

$$
\begin{aligned}
& \nabla_{X} \Omega=\alpha \theta_{1}(X) \Phi+\alpha \theta_{2}(X) \Psi \\
& \nabla_{X} \Phi=-\alpha \theta_{1}(X) \Omega+p(X) \Psi \\
& \nabla_{X} \Psi=-\alpha \theta_{2}(X) \Omega-p(X) \Phi .
\end{aligned}
$$

Consequently, using (2.2) we get

(2.3b) $\quad g\left(R\left(E_{1}, E_{4}\right) . J X, Y\right)=-\nabla_{\left[E_{1}, E_{4}\right]} \Omega-E_{4} \alpha \Phi-\alpha p\left(E_{4}\right) \Psi$,

(2.3c) $g\left(R\left(E_{2}, E_{3}\right) . J X, Y\right)=-\nabla_{\left[E_{2}, E_{3}\right]} \Omega+E_{3} \alpha \Psi-\alpha p\left(E_{3}\right) \Phi$,

(2.3d) $g\left(R\left(E_{4}, E_{2}\right) . J X, Y\right)=-\nabla_{\left[E_{4}, E_{2}\right]} \Omega-E_{4} \alpha \Psi+\alpha p\left(E_{4}\right) \Phi$,

(2.3e) $\quad g\left(R\left(E_{1}, E_{2}\right) . J X, Y\right)$

$$
=-\nabla_{\left[E_{1}, E_{2}\right]} \Omega+\left(E_{1} \alpha-\alpha p\left(E_{2}\right)\right) \Psi-\left(\alpha p\left(E_{1}\right)+E_{2} \alpha\right) \Phi,
$$

where as usual $R(X, Y) . J=\nabla_{X}\left(\nabla_{Y} J\right)-\nabla_{Y}\left(\nabla_{X} J\right)-\nabla_{[X, Y]} J$. Recall that

$$
R(X, Y) . J=R(X, Y) \circ J-J \circ R(X, Y),
$$

i.e. $R(X, Y)$ acts on the tensor $J$ as a derivation. Since $\mathcal{R}(L M) \subset \bigwedge^{+} M$ it is clear that

$$
\begin{aligned}
& g\left(R\left(E_{1}, E_{3}\right) . J X, Y\right)=g\left(R\left(E_{4}, E_{2}\right) . J X, Y\right), \\
& g\left(R\left(E_{3}, E_{2}\right) . J X, Y\right)=g\left(R\left(E_{4}, E_{1}\right) . J X, Y\right) .
\end{aligned}
$$

Consequently, from (2.3) and (2.4), using the condition $\mathcal{R}(L M) \subset \wedge^{+} M$, we get

(2.5a) $\quad \frac{1}{2} \mathcal{R}(\Phi, \Psi)=-g\left(R\left(E_{1}, E_{3}\right) . J E_{1}, E_{3}\right)=E_{3} \alpha+\alpha \theta_{1}\left(\left[E_{1}, E_{3}\right]\right)$

$$
=E_{3} \alpha-\alpha \Gamma_{11}^{3}
$$

$(2.5 \mathrm{~b}) \quad \frac{1}{2} \mathcal{R}(\Phi, \Psi)=g\left(R\left(E_{2}, E_{3}\right) . J E_{3}, E_{2}\right)=-\left(E_{3} \alpha+\alpha \theta_{2}\left(\left[E_{2}, E_{3}\right]\right)\right)$

$$
=-E_{3} \alpha+\alpha \Gamma_{22}^{3}
$$

$(2.5 \mathrm{c}) \quad \frac{1}{2} \mathcal{R}(\Phi, \Phi)=-g\left(R\left(E_{4}, E_{2}\right) . J E_{3}, E_{2}\right)=E_{4} \alpha-\alpha \theta_{2}\left(\left[E_{4}, E_{2}\right]\right)$

$$
=E_{4} \alpha-\alpha \Gamma_{22}^{4},
$$

$(2.5 \mathrm{~d}) \quad \frac{1}{2} \mathcal{R}(\Psi, \Psi)=-g\left(R\left(E_{1}, E_{4}\right) . J E_{1}, E_{3}\right)=-\left(-E_{4} \alpha-\alpha \theta_{1}\left(\left[E_{1}, E_{4}\right]\right)\right)$

$$
=E_{4} \alpha-\alpha \Gamma_{11}^{4} \text {. }
$$


(2.5e) $\quad \frac{1}{2} \mathcal{R}(\Phi, \Psi)=-g\left(R\left(E_{1}, E_{4}\right) . J E_{1}, E_{4}\right)=-\left(\alpha p\left(E_{4}\right)+\alpha \theta_{2}\left(\left[E_{1}, E_{4}\right]\right)\right)$

$$
=\alpha \Gamma_{44}^{3}-\alpha \Gamma_{14}^{2}
$$

$$
\begin{aligned}
& \frac{1}{2} \mathcal{R}(\Phi, \Psi)=-g\left(R\left(E_{4}, E_{2}\right) . J E_{1}, E_{3}\right)=\alpha p\left(E_{4}\right)+\alpha \theta_{1}\left(\left[E_{4}, E_{2}\right]\right) \\
& =-\alpha \Gamma_{24}^{1}-\alpha \Gamma_{44}^{3} \\
& (2.5 \mathrm{~g}) \quad \frac{1}{2} \mathcal{R}(\Phi, \Phi)=g\left(R\left(E_{1}, E_{3}\right) . J E_{1}, E_{4}\right)=-\alpha \theta_{2}\left(\left[E_{1}, E_{3}\right]\right)-\alpha p\left(E_{3}\right) \\
& =-\alpha \Gamma_{13}^{2}-\alpha \Gamma_{33}^{4}, \\
& \text { (2.5a) } \quad \frac{1}{2} \mathcal{R}(\Psi, \Psi)=-g\left(R\left(E_{2}, E_{3}\right) . J E_{1}, E_{3}\right)=\alpha \theta_{1}\left(\left[E_{1}, E_{3}\right]\right)-\alpha p\left(E_{3}\right) \\
& =\alpha \Gamma_{23}^{1}-\alpha \Gamma_{33}^{4} \text {. }
\end{aligned}
$$

Note that for a Lee form $\theta$ of $(M, g, J)$ we have $\theta=-2 \alpha \theta_{4}$. Write $X=2 \alpha E_{3}$. Then $d \Omega=-2 \alpha \theta_{4} \wedge \Omega$ and

$$
L_{X} \Omega=d\left(i_{X} \Omega\right)+i_{X}(d \Omega)=2 d\left(\alpha \theta_{4}\right)=-d \theta .
$$

Hence

$$
L_{X} \Omega^{2}=2 L_{X} \Omega \wedge \Omega=-2 d \theta \wedge \Omega .
$$

Since $d \Omega=\theta \wedge \Omega$ we have $d \theta \wedge \Omega=0$. Thus $L_{X} \Omega^{2}=0$ and $\operatorname{div} X=0$. This means that

$$
\Gamma_{11}^{3}+\Gamma_{22}^{3}+\Gamma_{44}^{3}=E_{3} \ln \alpha .
$$

From Lemma B, (2.5) and (2.6) we get (a)-(c) of Lemma C. Since $\theta=$ $-\delta \Omega \circ J$ we have $\nabla J\left(E_{1}, E_{1}\right)=\nabla J\left(E_{2}, E_{2}\right)=\alpha E_{3}$ and $\nabla J\left(E_{3}, E_{3}\right)=$ $\nabla J\left(E_{4}, E_{4}\right)=0$. Consequently,

$$
\alpha E_{3}+J\left(\nabla_{E_{1}} E_{1}\right)=\nabla_{E_{1}} E_{2}, \quad \alpha E_{3}+J\left(\nabla_{E_{2}} E_{2}\right)=-\nabla_{E_{2}} E_{1} .
$$

Thus $\Gamma_{12}^{3}+\Gamma_{11}^{4}=\alpha$ and $-\Gamma_{21}^{3}+\Gamma_{22}^{4}=\alpha$ and (d) follows. On the other hand $\mathcal{R}(\Phi, \Phi)+\mathcal{R}(\Psi, \Psi)=4\left(E_{4} \alpha-\alpha \Gamma_{11}^{4}\right)=4\left(\alpha \Gamma_{23}^{1}-\alpha \Gamma_{33}^{4}\right)$ and (e) follows.

Let us recall that Apostolov and Gauduchon [A-G-1] proved that a Hermitian surface with $J$-invariant Ricci tensor has symmetric $*$-Ricci tensor (equivalently $\Omega$ is an eigenfield of $W^{+}$). It follows that every Hermitian surface with $J$-invariant Ricci tensor satisfies condition $\left(G_{3}\right)$ of Gray. Recall also that the Ricci tensor $\varrho$ of $(M, g)$ is $J$-invariant if and only if $\mathcal{R}(L M) \subset \wedge^{+} M$. Note that Lemma $\mathrm{D}$ below can also be deduced from [De-1] and [A-G-1] if we additionally assume that $(M, g, J)$ is l.c.K. (locally conformally Kähler - since the nullity foliation is then spanned by a holomorphic Killing vector field $\xi$ and $J \xi$ and clearly $[\xi, J \xi]=0)$. In the compact case $(M, g, J)$ is l.c.K. but in general it may not be l.c.K.

Lemma D. Let $(M, g, J)$ be a Hermitian 4-dimensional manifold whose curvature tensor $\mathcal{R}$ satisfies $\mathcal{R}(L M) \subset \wedge^{+} M$. Then the Kähler form $\Omega$ of $(M, g, J)$ is an eigenform of the Weyl positive tensor $W^{+}$, i.e. $W^{+} \Omega=\lambda \Omega$ 
for $\lambda \in C^{\infty}(M)$ (or equivalently $(M, g, J)$ has symmetric $*$-Ricci tensor) and the nullity distribution $\mathcal{D}$ is involutive.

Proof. Note that it is enough to prove the lemma for $\left(M_{0}, g, J\right)$. Thus we can assume that $\mathcal{D}$ is a 2 -dimensional $J$-invariant distribution. Let $\left\{E_{3}, E_{4}\right\}$ be a local orthonormal basis in $\mathcal{D}$ such that $E_{4}=J E_{3}$. Hence

$$
\begin{aligned}
& \nabla_{E_{3}} J=0, \\
& \nabla_{E_{4}} J=0 .
\end{aligned}
$$

Consequently, we obtain

$$
\begin{aligned}
& \nabla_{E_{4} E_{3}}^{2} J+\nabla_{\nabla_{E_{4}} E_{3}} J=0, \\
& \nabla_{E_{3} E_{4}}^{2} J+\nabla_{\nabla_{E_{3}} E_{4}} J=0 .
\end{aligned}
$$

Thus $\nabla_{E_{3} E_{4}}^{2} J-\nabla_{E_{4} E_{3}}^{2} J+\nabla_{\left[E_{3}, E_{4}\right]} J=0$. Hence

$$
R\left(E_{3}, E_{4}\right) . J=-\nabla_{\left[E_{3}, E_{4}\right]} J .
$$

Choose a local orthonormal basis $\left\{E_{1}, E_{2}\right\}$ of $\mathcal{D}^{\perp}$ such that $J E_{1}=E_{2}$ and (2.1) holds. From (2.7) we obtain

$$
R\left(E_{3}, E_{4}, J X, Y\right)+R\left(E_{3}, E_{4}, X, J Y\right)=-\nabla_{\left[E_{3}, E_{4}\right]} \Omega(X, Y) .
$$

Consequently,

$$
\begin{aligned}
\mathcal{R}\left(E_{3} \wedge E_{4}, E_{2} \wedge E_{3}+E_{1} \wedge E_{4}\right) & =\mathcal{R}\left(E_{3} \wedge E_{4}, \Psi\right) \\
& =\alpha \theta_{1}\left(\left[E_{3}, E_{4}\right]\right), \\
\mathcal{R}\left(E_{3} \wedge E_{4}, E_{1} \wedge E_{3}-E_{2} \wedge E_{4}\right) & =\mathcal{R}\left(E_{3} \wedge E_{4}, \Phi\right) \\
& =\alpha \theta_{2}\left(\left[E_{3}, E_{4}\right]\right) .
\end{aligned}
$$

Set $a=\mathcal{R}\left(E_{3} \wedge E_{4}, \Psi\right), b=\mathcal{R}\left(E_{3} \wedge E_{4}, \Phi\right), c=\mathcal{R}\left(E_{1} \wedge E_{2}, \Psi\right), d=$ $\mathcal{R}\left(E_{1} \wedge E_{2}, \Phi\right)$. Note that the form $\bar{\Omega}=E_{1} \wedge E_{2}-E_{3} \wedge E_{4}$ is anti-self-dual $\left(\bar{\Omega} \in \bigwedge^{-} M\right)$. Thus $c-a=0=d-b$. We also have $\mathcal{R}(\Omega, \Phi)=b+d$, $\mathcal{R}(\Omega, \Psi)=a+c$. Consequently,

$$
\mathcal{R}(\Omega, \Phi)=2 b=2 \alpha \theta_{2}\left(\left[E_{3}, E_{4}\right]\right), \quad \mathcal{R}(\Omega, \Psi)=2 a=2 \alpha \theta_{1}\left(\left[E_{3}, E_{4}\right]\right) .
$$

It is clear that $\Omega$ is an eigenform of $W^{+}$if and only if $\mathcal{R}(\Omega, \Phi)=0, \mathcal{R}(\Omega, \Psi)$ $=0$. The last two equations are equivalent to the symmetry of the $*$-Ricci tensor (it also means that the component $W_{2}^{+}$of the positive Weyl tensor vanishes). Note also that $\mathcal{D}$ is a minimal foliation.

The first part of the next lemma is well known (see [A-G-1]).

Lemma E. Let $(M, g, J)$ be a Hermitian surface with J-invariant Ricci tensor. Then $\Gamma_{13}^{4}=-E_{2} \ln \alpha, \Gamma_{23}^{4}=E_{1} \ln \alpha$ and $d \theta$ is anti-self-dual. In particular if $M$ is compact then $(M, g, J)$ is locally conformally Kähler. In addition $(M, g, J)$ is l.c.K. if and only if $E_{3} \alpha=0, \Gamma_{34}^{1}=E_{2} \ln \alpha, \Gamma_{34}^{2}=$ $-E_{1} \ln \alpha$. 
Proof. From [A-G-1, Th. 2] it follows that $\Omega$ is an eigenform of $W^{+}$. Let $\left\{E_{1}, E_{2}, E_{3}, E_{4}\right\}$ be a local special frame. From (2.3e) we deduce that the equation $\mathcal{R}(\Omega, \Phi)=0$ is equivalent to $-\alpha \theta_{1}\left(\left[E_{1}, E_{2}\right]\right)-\left(\alpha p\left(E_{1}\right)+E_{2} \alpha\right)=0$. Analogously the equation $\mathcal{R}(\Omega, \Psi)=0$ is equivalent to $-\alpha \theta_{2}\left(\left[E_{1}, E_{2}\right]\right)-$ $\alpha p\left(E_{2}\right)+E_{1} \alpha=0$. We get $\Gamma_{13}^{4}=-E_{2} \ln \alpha, \quad \Gamma_{23}^{4}=E_{1} \ln \alpha$ after some easy computation. Note that

(2.13a) $d \theta_{4}\left(E_{3}, E_{4}\right)=-\theta_{4}\left(\left[E_{3}, E_{4}\right]\right)=-E_{3} \ln \alpha$,

(2.13b) $d \theta_{4}\left(E_{1}, E_{2}\right)=-\theta_{4}\left(\left[E_{1}, E_{2}\right]\right)=-\Gamma_{12}^{4}+\Gamma_{21}^{4}=-2 E_{3} \ln \alpha$,

(2.13c) $d \theta_{4}\left(E_{1}, E_{3}\right)=-\theta_{4}\left(\left[E_{1}, E_{3}\right]\right)=-\Gamma_{13}^{4}+\Gamma_{31}^{4}$,

(2.13d) $d \theta_{4}\left(E_{2}, E_{3}\right)=-\theta_{4}\left(\left[E_{2}, E_{3}\right]\right)=-\Gamma_{23}^{4}+\Gamma_{32}^{4}$.

We also have $d \theta=-2 d \alpha \wedge \theta_{4}-2 \alpha d \theta_{4}$. From (2.13) we get

$$
-\frac{1}{2} d \theta=2 E_{3} \alpha \bar{\Omega}+\left(-\alpha \Gamma_{34}^{1}+E_{2} \alpha\right) \bar{\Phi}+\left(-\alpha \Gamma_{32}^{4}+E_{1} \alpha\right) \bar{\Psi}
$$

where $\bar{\Phi}=\theta_{1} \wedge \theta_{3}+\theta_{2} \wedge \theta_{4}, \bar{\Psi}=\theta_{1} \wedge \theta_{4}-\theta_{2} \wedge \theta_{3}$. Consequently, $d \theta$ is anti-self-dual.

If $(M, g, J)$ is a Hermitian surface with $|\nabla J| \neq 0$ on $M$ then the distributions $\mathcal{D}, \mathcal{D}^{\perp}$ define a natural opposite almost Hermitian structure $\bar{J}$ on $M$. This structure is defined as follows: $\left.\bar{J}\right|_{\mathcal{D}}=-\left.J\right|_{\mathcal{D}},\left.\bar{J}\right|_{\mathcal{D}^{\perp}}=\left.J\right|_{\mathcal{D}^{\perp}}$. In the special basis we just have $\bar{J} E_{1}=E_{2}, \bar{J} E_{3}=-E_{4}$.

Lemma F. Let $(M, g, J)$ be a Hermitian l.c.K. 4-manifold with Hermitian Ricci tensor. Assume that $|\nabla J| \neq 0$ on $M$. Then the following conditions are equivalent:

(a) $(M, g, \bar{J})$ is a Hermitian surface.

(b) $\nabla \alpha \| E_{4}=-\frac{1}{2 \alpha} \theta^{\sharp}$.

(c) $\mathcal{D}$ is a totally geodesic foliation.

(d) $\mathcal{D}$ is contained in the nullity of $\bar{J}$.

(e) $\nabla_{E_{4}} E_{4}=0$ (equivalently $\nabla_{\theta^{\sharp}} \theta^{\sharp} \wedge \theta^{\sharp}=0$ ).

(f) $d|\theta|^{2} \wedge \theta=0$.

Proof. Choose a local orthonormal frame $\left\{E_{1}, \ldots, E_{4}\right\}$ such that (2.1) holds. Since $(M, g, J)$ is l.c.K. we have $d \theta=0$ and consequently

$$
E_{3} \ln \alpha=0, \quad \Gamma_{34}^{1}=E_{2} \ln \alpha, \quad \Gamma_{34}^{2}=-E_{1} \ln \alpha .
$$

From the equalities $J\left(\nabla_{E_{3}} E_{3}\right)=\nabla_{E_{3}} E_{4}$ and $J\left(\nabla_{E_{4}} E_{4}\right)=-\nabla_{E_{4}} E_{3}$ we obtain

$$
-\Gamma_{33}^{1}=\Gamma_{44}^{1}=-\Gamma_{34}^{2}=E_{1} \ln \alpha, \quad \Gamma_{44}^{2}=\Gamma_{34}^{1}=-\Gamma_{33}^{2}=E_{2} \ln \alpha .
$$

Note that (we write $\nabla_{X} \theta_{i}=\omega_{i}^{j}(X) \theta_{j}, \bar{\Phi}=\theta_{1} \wedge \theta_{3}+\theta_{2} \wedge \theta_{4}, \bar{\Psi}=\theta_{1} \wedge \theta_{4}-$ $\left.\theta_{2} \wedge \theta_{3}\right)$

$\nabla\left(\theta_{1} \wedge \theta_{2}\right)=\frac{1}{2}\left\{\Phi\left(\omega_{1}^{4}+\omega_{2}^{3}\right)+\Psi\left(\omega_{3}^{1}+\omega_{2}^{4}\right)+\bar{\Phi}\left(-\omega_{1}^{4}+\omega_{2}^{3}\right)+\bar{\Psi}\left(-\omega_{3}^{1}+\omega_{2}^{4}\right)\right\}$. 
Analogously

$\nabla\left(\theta_{3} \wedge \theta_{4}\right)=\frac{1}{2}\left\{\Phi\left(\omega_{1}^{4}+\omega_{2}^{3}\right)+\Psi\left(\omega_{3}^{1}+\omega_{2}^{4}\right)-\bar{\Phi}\left(-\omega_{1}^{4}+\omega_{2}^{3}\right)-\bar{\Psi}\left(-\omega_{3}^{1}+\omega_{2}^{4}\right)\right\}$.

Note that $\nabla \Omega=a \otimes \Phi+b \otimes \Psi$ and $\nabla \bar{\Omega}=a^{\prime} \otimes \bar{\Phi}+b^{\prime} \otimes \bar{\Psi}$ where under our assumptions $a=\alpha \theta_{1}$ and $b=-\alpha \theta_{2}$. It is clear that $(M, g, \bar{J})$ is Hermitian if and only if

$$
a^{\prime}=b^{\prime} \circ J
$$

On the other hand $a=\omega_{1}^{4}+\omega_{2}^{3}, b=\omega_{3}^{1}+\omega_{2}^{4}$ and

$$
\begin{aligned}
\alpha \theta_{1} & =\omega_{1}^{4}+\omega_{2}^{3}, & -\alpha \theta_{2} & =\omega_{3}^{1}+\omega_{2}^{4}, \\
a^{\prime} & =-\omega_{1}^{4}+\omega_{2}^{3}, & b^{\prime} & =\omega_{3}^{1}-\omega_{2}^{4} .
\end{aligned}
$$

It is clear from (2.15), (2.16) that $\mathcal{D}$ is in the nullity of $\bar{J}$ if and only if $E_{1} \alpha=E_{2} \alpha=0$. The last condition is also equivalent to $\mathcal{D}$ being totally geodesic. Recall that $\Gamma_{k j}^{i}=\omega_{j}^{i}\left(E_{k}\right)$. It is also clear from (2.17) that $(M, g, \bar{J})$ is Hermitian if and only if (b) holds. Since $\Gamma_{44}^{1}=E_{1} \ln \alpha, \Gamma_{44}^{2}=E_{2} \ln \alpha$, $\Gamma_{44}^{3}=-E_{3} \ln \alpha$, (e) is equivalent to (b). Note that $|\theta|^{2}=4 \alpha^{2}$, thus (f) is equivalent to $(\mathrm{b})$.

Lemma G. Let $(M, g, J)$ be a Hermitian 4-manifold. Assume that $|\nabla J|$ $\neq 0$ on $M$. If $(M, g, J)$ has Hermitian Ricci tensor then $d \bar{\Omega}=2\left(\Gamma_{12}^{3}-\right.$ $\left.\Gamma_{22}^{4}\right) \theta_{4} \wedge \theta_{1} \wedge \theta_{2}$. If $\delta W^{+}=0$ and $\left|W^{+}\right|$is non-vanishing on $M$ then $\alpha=$ $\frac{1}{3} E_{4} \ln |\kappa|$ (equivalently $2 \alpha^{2}=-\frac{1}{3} \theta^{\sharp} \ln |\kappa|$ ) and if $(M, g)$ is Einstein then $\nabla \tau^{*} \| E_{4}$.

Proof. Note that from the Cartan structure equations, $d \theta_{i}=-\sum_{p=1}^{4} \omega_{p}^{i}$ $\wedge \theta_{p}$. Hence using the above results we obtain

$$
\begin{aligned}
& d\left(\theta_{1} \wedge \theta_{2}\right)=-2 \Gamma_{11}^{4} \theta_{4} \wedge \theta_{1} \wedge \theta_{2}, \\
& d\left(\theta_{3} \wedge \theta_{4}\right)=-2 \Gamma_{12}^{3} \theta_{4} \wedge \theta_{1} \wedge \theta_{2} .
\end{aligned}
$$

Thus

$$
d \bar{\Omega}=2\left(\Gamma_{12}^{3}-\Gamma_{22}^{4}\right) \theta_{4} \wedge \theta_{1} \wedge \theta_{2} .
$$

Now assume that $\delta W^{+}=0$ and $\left|W^{+}\right| \neq 0$ on $M$. Then (see [A-G-1, p. 431]) we have

$$
\theta=-\frac{2}{3} d \ln |\kappa|=-d f
$$

where $f=\frac{2}{3} \ln \left|3 \tau^{*}-\tau\right|$. Note that since $\theta=-2 \alpha \theta_{4}$ we get $E_{1} f=E_{2} f=$ $E_{3} f=0$. Consequently, $\theta=-2 \alpha \theta_{4}=-E_{4} f \theta_{4}$ and $2 \alpha=E_{4} f$. If $(M, g)$ is Einstein then $\delta W=0$ and either $\kappa=0$ on $M$ or $\left|W^{+}\right| \neq 0$ on $M$. Since $\tau$ is constant it follows that in the first case $\tau^{*}=\frac{1}{3} \tau$ is constant, while $E_{1} \tau^{*}=E_{2} \tau^{*}=E_{3} \tau^{*}=0$ in the second case.

Recall that an Einstein-Hermitian 4-manifold is l.c.K. unless it is ASD, i.e. $W^{+}=0$ (see $[$ De-1] and $[\mathrm{A}-\mathrm{G}-1]$ ). 
Corollary. Let $(M, g, J)$ be an Einstein-Hermitian 4-manifold which is not $A S D$. Assume that $|\nabla J| \neq 0$ on $M$. Then the following conditions are equivalent:

(a) $(M, g, \bar{J})$ is a Hermitian surface,

(b) $\Delta \theta=\lambda \theta$ for some $\lambda \in C^{\infty}(M)$.

Proof. From (1.6) it follows that $d \kappa=-\frac{3}{2}\left(d|\theta|^{2}+2 \Delta \theta\right)$. Since $d \kappa=-\frac{2}{3} \kappa \theta$ we get $d|\theta|^{2} \wedge \theta=-2 \Delta \theta \wedge \theta$ and the result is a consequence of Lemma $\mathrm{F}(\mathrm{f})$.

Lemma H. Let $(M, g, J)$ be a Hermitian 4-manifold with Hermitian Ricci tensor. If $\delta W^{+}=0$ and $\left|W^{+}\right|$is non-vanishing on $M$ then $\kappa \neq 0$ on $M$ and the field $X=J\left(\nabla \kappa^{-1 / 3}\right)$ is a holomorphic Killing vector field for $(M, g, J)$. What is more, $X=\frac{1}{2} \kappa^{-1 / 3} J\left(\theta^{\sharp}\right)$ and $|X|=\alpha \kappa^{-1 / 3}$. In particular the set $\alpha^{-1}(0)$ is a totally geodesic submanifold of $(M, g)$.

Proof. The first statement can be proved analogously to [De-1, Prop. 4 and Prop. 5] and [A-G-1, Prop. 1]). Note that

$$
X=J\left(\nabla \kappa^{-1 / 3}\right)=-\frac{1}{3} J(\nabla \kappa) \kappa^{-4 / 3}=-\frac{1}{3} J(\nabla \ln |\kappa|) \kappa^{-1 / 3}=\frac{1}{2} \kappa^{-1 / 3} J\left(\theta^{\sharp}\right) .
$$

Since $\theta^{\sharp}=-2 \alpha E_{4}$ we get $|X|=\alpha \kappa^{-1 / 3}$.

Since $X$ is a holomorphic vector field and $X_{x}=0$ if and only if $\alpha(x)=0$ we obtain

Corollary. Let $(M, g, J)$ be a Hermitian non-Kähler 4-manifold with Hermitian Ricci tensor. If $\delta W^{+}=0$ and $\left|W^{+}\right|$is non-vanishing on $M$ then the set $F=\left\{x \in M:\left|\nabla J_{x}\right|=0\right\}$ is nowhere dense (i.e. $U=M-F$ is an open dense subset of $M$ ).

REMARK. It is easy to see exactly as above that also the following statement holds:

Let $(M, g, J)$ be a Hermitian non-Kähler 4-manifold with Hermitian Ricci tensor. Assume that $(M, g, J)$ is conformally Kähler and let $A$ be a smooth function such that $d A=\frac{1}{2} \theta$. Thus $(M, \bar{g}, J)$ is Kähler where $\bar{g}=$ $\exp (-2 A) g$. Then the field $X=J(\bar{\nabla} \exp (-A))=e^{A} J(\nabla A)=\frac{1}{2} e^{A}(\delta \Omega)^{\sharp}$ is a holomorphic Killing vector field for $(M, g, J)$ and $|X|=e^{A} \alpha$. The set $F=\left\{x \in M:\left|\nabla J_{x}\right|=0\right\}$ is totally geodesic and nowhere dense.

Lemma I. Let $(M, g, J)$ be a compact Hermitian Einstein non-Kähler 4-manifold. Assume that the natural opposite almost Hermitian structure $\bar{J}$ defined on the set $U=M-\alpha^{-1}(0)$ is Hermitian. Then $\bar{J}$ extends smoothly to a global opposite Hermitian structure $\bar{J}$ on $M$.

Proof. Since $(M, g)$ is Einstein it follows that either $W^{-}=0$ or $W^{-} \neq 0$ everywhere. From our assumptions it follows that the scalar curvature $\tau$ of $(M, g)$ is positive (see [G-M, Th. 1.1] and [C-S-V, Th. 2.1] or [LeB]). Thus in view of $[\mathrm{H}]$ and $[\mathrm{A}-\mathrm{D}-\mathrm{M}]$ we get $W^{-} \neq 0$ on $M$. On the open dense subset $U$ 
the tensor $W^{-}$has exactly two eigenvalues. Since $\operatorname{tr} W^{-}=0$ and $W^{-} \neq 0$ everywhere it is clear that $W^{-}$has two eigenvalues everywhere. The Kähler form $\bar{\Omega}$ of $(U, g, \bar{J})$ is a simple eigenform of $\left.W^{-}\right|_{U}$. In view of the above results it extends to a global simple eigenform of $W^{-}$.

For the description of D. Page's Einstein-Hermitian metric on $\mathbb{C} P^{2} \sharp$ $\overline{\mathbb{C} P^{2}}$ we refer to $[\mathrm{K}]$ (see also $[\mathrm{B}]$ ). The Hermitian structure on $\mathbb{C} P^{2} \sharp \overline{\mathbb{C} P^{2}}$ is given by $J H=X / f, J Y=Z$ where $X, Y, Z$ are left invariant vector fields on $\mathrm{SU}(2)=S^{3}$ and the metric on the open dense subset $U=$ $(0, l) \times S^{3}$ of $\mathbb{C} P^{2} \sharp \overline{\mathbb{C} P^{2}}$ is given by $g=d t^{2}+g_{t}$ where $g_{t}=f(t)^{2} \theta_{1}^{2}+$ $g(t)^{2}\left(\theta_{2}^{2}+\theta_{3}^{2}\right)$ where $\left\{\theta_{1}, \theta_{2}, \theta_{3}\right\}$ is the left invariant co-frame on $\mathrm{SU}(2)$ dual to the frame $\{X, Y, Z\}$. It is easy to show using the O'Neill formulas that $\nabla J(H, H)=0$, which means that $H \in \mathcal{D}$. We also have $\delta \Omega \|$ $X$. Hence we obtain (in Koda's notation) $\mathcal{D}=\operatorname{span}\{H, X\}$ and $E_{4}=$ $H$. Since $\nabla_{H} H=0$ it follows that the natural opposite almost Hermitian structure $\bar{J}$ given by $\bar{J} H=-X / f, \bar{J} Y=Z$ is Hermitian. Clearly this structure extends to a global opposite Hermitian structure on $\mathbb{C} P^{2} \sharp$ $\overline{\mathbb{C} P^{2}}$. From $[\mathrm{LeB}]$ it follows that an Eistein-Hermitian non-Kähler surface $(M, g)$ is a blow-up of $\mathbb{C} P^{2}$ at one, two or three points in general position. C. LeBrun also proves that the Einstein-Hermitian metric on $\mathbb{C} P^{2} \sharp \overline{\mathbb{C} P^{2}}$ is uniquely determined up to isometry. It follows that the only compact Einstein-Hermitian surface with opposite Hermitian structure is isometrically biholomorphic to $\mathbb{C} P^{2} \sharp \overline{\mathbb{C} P^{2}}$ with D. Page's metric. Indeed, if $(M, g, J)$ is a compact Einstein surface then it satisfies $\tau(M) \leq 0$ as a blow-up of $\mathbb{C} P^{2}$ where $\tau(M)$ denotes the signature of $(M, g, J)$. Since $(M, g, \bar{J})$ is also a compact Hermitian surface we have $\tau(M, \bar{J})=-\tau(M, J) \leq 0$. Consequently, $\tau(M)=0$ and $(M, g, J)$ must be isometrically biholomorphic to $\mathbb{C} P^{2} \sharp \overline{\mathbb{C} P^{2}}$ with D. Page's metric. Hence we have from [LeB] and Lemma I:

Proposition 2. Let $(M, g, J)$ be a compact Einstein-Hermitian nonKähler 4-manifold. Assume that the natural opposite almost Hermitian structure $\bar{J}$ defined on the set $U=\left\{x \in M:\left|\nabla J_{x}\right| \neq 0\right\}$ is Hermitian. Then $(M, g, J)$ is isometrically biholomorphic to $\mathbb{C} P^{2} \sharp \overline{\mathbb{C} P^{2}}$ with D. Page's metric.

3. Einstein-Hermitian metrics of cohomogeneity 1. Our present aim is to show how to construct all non-compact examples of Hermitian surfaces with Hermitian natural opposite structure. Since an Einstein-Hermitian non-Kähler manifold $M$ satisfies the condition $\nabla J \neq 0$ on an open dense subset of $M$ we shall assume that a non-Kähler Einstein-Hermitian manifold $M$ satisfies $\nabla J \neq 0$ on the whole of $M$. Recall also that a homo- 
geneous Einstein 4-dimensional manifold is locally symmetric and that an Einstein-Hermitian surface $(M, g, J)$ satisfies one of the two conditions: (1) $(M, g)$ is ASD, i.e. $W^{+}=0$, or $(2) W^{+} \neq 0$ on the whole of $M$ (see [De-1], [A-G-1]). We shall prove

Theorem 1. Let $(M, g, J)$ be an Einstein-Hermitian non-Kähler 4manifold. Assume that $W^{+}$is non-vanishing on $M$. Then the following conditions are equivalent:

(a) $(M, g)$ is (locally) of cohomogeneity 1 ,

(b) $(M, g, \bar{J})$ is a Hermitian surface,

(c) $M$ is locally isometric to the manifold $\widetilde{M}=\mathbb{R} \times P_{0}$, where $\left(P_{0}, g_{0}\right)$ is a 3-dimensional naturally reductive manifold (the total space of a Riemannian submersion $p: P_{0} \rightarrow M_{0}$ over a Riemannian surface $M_{0}$ of constant sectional curvature $K \in\{-1,0,1\})$ with a metric

$$
g=d t^{2}+a(t)^{2} \theta^{2}+b(t)^{2} p^{*} g_{\text {can }},
$$

where $g_{0}=\theta^{2}+p^{*} g_{\text {can }}, \theta$ is the connection form of $P_{0}$ such that $p^{*} d \theta=$ $c \mathrm{vol}_{\text {can }}, c \in \mathbb{R}$ and $\mathrm{vol}_{\text {can }}$ is the volume form of the canonical metric on $M_{0}$.

Proof. We shall prove the implications (b) $\Rightarrow(\mathrm{c}) \Rightarrow(\mathrm{a}) \Rightarrow(\mathrm{b})$. We start with the proof of $(\mathrm{b}) \Rightarrow(\mathrm{c})$. Note that $\bar{J}$ is a global opposite Hermitian structure on $M$. Let $\left\{E_{1}, E_{2}, E_{3}, E_{4}\right\}$ be a local standard frame in $U$. Denote by $\bar{\tau}^{*}$ the $*$-scalar curvature of $(M, g, \bar{J})$. Also write $\beta=\frac{1}{2 \sqrt{2}}|\nabla \bar{J}|$. Then $d \bar{\Omega}=$ $-2 \varepsilon \beta \theta_{4} \wedge \theta_{1} \wedge \theta_{2}$ and $\delta \bar{\Omega}=2 \varepsilon \beta E_{3}, \bar{\theta}=-2 \varepsilon \beta \theta_{4}$ where $\varepsilon \in\{-1,1\}, \mathcal{D}=\{X$ : $\left.i_{X}(\Omega-\bar{\Omega})=0\right\}$. Consequently, the distribution $\mathcal{D}^{\perp}$ on $U$ also extends to a globally defined distribution. From Lemma $\mathrm{F}$ it follows that $\nabla_{E_{4}} E_{4}=0$, $\nabla \alpha \| E_{4}$ and the foliation $\mathcal{D}$ is totally geodesic. Since $\mathcal{R}(\Omega, \Omega)=\frac{1}{2} \tau^{*}$ and $\mathcal{R}(\bar{\Omega}, \bar{\Omega})=\frac{1}{2} \bar{\tau}^{*}$ we get $R_{1212}+R_{3434}=-\frac{1}{4}\left(\tau^{*}+\bar{\tau}^{*}\right)$. Since $(M, g)$ is an Einstein space we have $R_{1212}=R_{3434}$. Thus we get $K_{\mathcal{D}}=-R_{3434}=$ $\frac{1}{8}\left(\tau^{*}+\bar{\tau}^{*}\right)$ where $K_{\mathcal{D}}$ denotes the sectional curvature of leaves of the foliation $\mathcal{D}$. Note that $\tau^{*}$ is non-constant, the distribution $S=\operatorname{span}\left\{E_{1}, E_{2}, E_{3}\right\}$ is involutive and its leaves are $M(c)=\tau^{*-1}(c)$. Choose local coordinates $\left(t, x_{1}, x_{2}, x_{3}\right)$ such that $E_{4}=\partial / \partial t$. Then $c=c(t)$ and we can parameterize $M(c)$ as $M(c(t))=M(t)$.

We shall show that every leaf of $S$ is a 3 -dimensional naturally reductive space $P_{0}$ which is the total space of a Riemannian submersion over a Riemannian surface of constant sectional curvature. Note that $P_{0}$ is one of the Lie groups $\mathrm{SU}(2), H, \mathrm{SL}(2, \mathbb{R})$ with a left invariant metric or is a Riemannian product $\mathbb{R} \times M_{0}$ where $H$ denotes the Heisenberg group and $M_{0}$ is a (real) surface of constant curvature $K \in\{-1,0,1\}$. In view of the results of Pedersen and Tod [P-T] it is enough to show that every leaf $\mathrm{S}$ of $S$ is an $\mathcal{A}$-manifold. 
Using the formula

$$
\begin{aligned}
R_{i j k s} & =g\left(R\left(E_{i}, E_{j}\right) E_{k}, E_{s}\right) \\
& =E_{i} \Gamma_{j k}^{s}-E_{j} \Gamma_{i k}^{s}+\Gamma_{j k}^{p} \Gamma_{i p}^{s}-\Gamma_{i k}^{p} \Gamma_{j p}^{s}-\left(\Gamma_{i j}^{p}-\Gamma_{j i}^{p}\right) \Gamma_{p k}^{s},
\end{aligned}
$$

in view of $E_{1} \alpha=E_{2} \alpha=E_{3} \alpha=0$ we obtain

$$
\begin{aligned}
& R_{1313}=R_{2424}=\Gamma_{11}^{4} \Gamma_{33}^{4}-\left(\Gamma_{12}^{3}\right)^{2}, \\
& R_{2323}=R_{1414}=\Gamma_{11}^{4} \Gamma_{33}^{4}-\left(\Gamma_{12}^{3}\right)^{2}, \\
& R_{3434}=R_{1212}=E_{4}^{2} \ln \alpha-E_{4} \alpha+\left(\Gamma_{33}^{4}\right)^{2}, \\
& R_{1234}=2 \Gamma_{11}^{4} \Gamma_{12}^{3}-2 \Gamma_{12}^{3} \Gamma_{33}^{4} .
\end{aligned}
$$

Denote by $R^{\mathrm{S}}$ the curvature tensor of the leaf $\mathrm{S}$ of foliation $S$. Then

$$
\begin{aligned}
g(R(X, Y) Z, W)= & g\left(R^{\mathrm{S}}(X, Y) Z, W\right)+g(A(X, Z), A(Y, W)) \\
& -g(A(Y, Z), A(X, W))
\end{aligned}
$$

where $A$ denotes the second fundamental form of the hypersurface $\mathrm{S}$. Since also $R\left(E_{3}, E_{4}\right) \bar{J}=0$ we obtain

$$
\begin{aligned}
R_{3413} & =R_{3424}=R_{3423}=R_{3424}=R_{1213} \\
& =R_{1224}=R_{1223}=R_{1224}=0 .
\end{aligned}
$$

It is easy to see that $\varrho^{\mathrm{S}}\left(E_{1}, E_{3}\right)=\varrho^{\mathrm{S}}\left(E_{2}, E_{3}\right)=\varrho^{\mathrm{S}}\left(E_{1}, E_{2}\right)=0$ where $\varrho^{\mathrm{S}}$ is the Ricci tensor of the hypersurface $\mathrm{S}$. We also have

$$
\begin{aligned}
& R_{1212}^{\mathrm{S}}=R_{1212}-\Gamma_{11}^{4} \Gamma_{22}^{4}=R_{1212}-\left(\Gamma_{11}^{4}\right)^{2}, \\
& R_{1313}^{\mathrm{S}}=R_{1313}-\Gamma_{11}^{4} \Gamma_{33}^{4}, \\
& R_{2323}^{\mathrm{S}}=R_{2323}-\Gamma_{22}^{4} \Gamma_{33}^{4} .
\end{aligned}
$$

Note that from Lemma $\mathrm{C}(\mathrm{d})$ and (2.19) we have

$$
\Gamma_{12}^{3}=\frac{\alpha-\varepsilon \beta}{2}, \quad \Gamma_{11}^{4}=\frac{\alpha+\varepsilon \beta}{2} .
$$

Consequently, the Ricci tensor of $\left(\mathrm{S},\left.g\right|_{\mathrm{S}}\right)$ has two eigenvalues $\lambda, \mu$ such that

$$
\begin{aligned}
\lambda & =\varrho^{\mathrm{S}}\left(E_{3}, E_{3}\right)=2\left(\Gamma_{12}^{3}\right)^{2}=2\left(\frac{\alpha-\varepsilon \beta}{2}\right)^{2}, \\
\mu & =\varrho^{\mathrm{S}}\left(E_{1}, E_{1}\right)=\varrho^{\mathrm{S}}\left(E_{2}, E_{2}\right) \\
& =\left(\Gamma_{11}^{4}\right)^{2}-\left(\Gamma_{33}^{4}\right)^{2}+E_{4} \alpha-E_{4}^{2} \ln \alpha+\left(\Gamma_{12}^{3}\right)^{2} \\
& =\left(\Gamma_{11}^{4}\right)^{2}+\frac{\tau^{*}+\bar{\tau}^{*}}{8}+\left(\Gamma_{12}^{3}\right)^{2} .
\end{aligned}
$$

It is clear that $\lambda, \mu$ are constant on every leaf $\mathrm{S}$ of the foliation $S$. We show that $\left(\mathrm{S},\left.g\right|_{\mathrm{S}}\right)$ is an $\mathcal{A}$-manifold. It is enough to show (see $[\mathrm{J}]$ ) that $\nabla_{E_{1}}^{\mathrm{S}} E_{1}, \nabla_{E_{2}}^{\mathrm{S}} E_{2}, \nabla_{E_{1}}^{\mathrm{S}} E_{2}+\nabla_{E_{2}}^{\mathrm{S}} E_{1} \in \mathcal{D}^{\perp}$ and $\nabla_{E_{3}}^{\mathrm{S}} E_{3}=0$ where $\nabla^{\mathrm{S}}$ is the induced Levi-Civita connection of $\left(\mathrm{S},\left.g\right|_{\mathrm{S}}\right)$. The above conditions are consequences of the equations $\Gamma_{11}^{3}=\Gamma_{22}^{3}=\Gamma_{12}^{3}+\Gamma_{21}^{3}=\Gamma_{33}^{1}=\Gamma_{33}^{2}=0$, which 
hold true in view of Lemma $\mathrm{C}$ and (2.16). It follows that every leaf $\left(\mathrm{S},\left.g\right|_{\mathrm{S}}\right)$ of $S$ is a 3 -dimensional $\mathcal{A}$-manifold. Note that it also means that the metric $f g$ of the foliated manifold $(M, \mathcal{D}, f g)$ is bundle-like for a positive function $f$ satisfying the equation $E_{4} \ln f=2 \Gamma_{11}^{4}=\alpha+\varepsilon \beta$. Note that the differential form $\omega=(\alpha+\varepsilon \beta) \theta_{4}$ is well defined and closed (note that $d \theta_{4}=0$ ), thus (we can assume $H^{1}(M, \mathbb{R})=0$ ) there exists a function $F \in C^{\infty}(M)$ such that $d F=\omega$. We take $f=\exp F$. It follows that the distribution $\mathcal{D}^{\perp}$ is geodesic in $(M, f g)$ (i.e. $\nabla_{X}^{f} X \in \Gamma\left(\mathcal{D}^{\perp}\right)$ if $X \in \Gamma\left(\mathcal{D}^{\perp}\right)$ where $\nabla^{f}$ is the Levi-Civita connection of $(M, f g)$ ), which means that $(M, f g, \mathcal{D})$ has a bundle-like metric. Thus (locally) $M$ is a locally trivial bundle over the space of leaves $M / \mathcal{D}$ and the natural projection $p: M \rightarrow M / \mathcal{D}$ is a Riemannian submersion (this is a consequence of $[\mathrm{M}]$ ), precisely for every $x_{0} \in M$ there exists a neighborhood $U$ of $x_{0}$ such that $p:(U, f g) \rightarrow\left(U / \mathcal{D}, g_{*}\right)$ is a Riemannian submersion onto the Riemannian manifold $\left(U / \mathcal{D}, g_{*}\right)$ with the induced Riemannian metric $g_{*}$.

It follows that the manifold $M(t)$ is isometric to a locally trivial bundle over $\left(M_{0}, b(t)^{2}\right.$ can) (where can denotes the standard metric on $M_{0}$ ), i.e. it is the total space of a Riemannian submersion $p: M(c) \rightarrow M_{0}$ with a metric

$$
g_{c}=a(t)^{2} \eta \otimes \eta+b(t)^{2} p^{*} \text { can, }
$$

where $\eta=\frac{1}{2 \alpha} \delta \Omega$ and $a(t), b(t)$ depend only on $t$. Note that the horizontal space of any such fibration coincides with $\mathcal{D}^{\perp}$. Thus $M$ has a metric

$$
g=d t^{2}+a(t)^{2} \eta \otimes \eta+b(t)^{2} p^{*} \text { can, }
$$

where $a, b$ are smooth functions depending on $t$. Note that $a=l_{0}|X|=$ $l_{0} \kappa^{-1 / 3} \alpha$ for some constant $l_{0} \in \mathbb{R}_{+}$. Every $\mathcal{A}$-manifold $M(t)$ with the metric $g_{t}=a(t)^{2} \eta \otimes \eta+b(t)^{2} p^{*}$ can admits a 3-dimensional group $G$ of isometries whose Lie algebra $\mathfrak{g}$ consists of lifts of Killing fields on $\left(M_{0}\right.$, can) (they correspond to the Killing fields on $G$ with any left invariant metric which are right invariant vector fields on $G$ ). Thus from (3.6) it is clear that $M$ admits a 3-dimensional group of isometries $G$ such that $G$ preserves every $M(t)$ and $[X, Y]=0$ (where $X=J\left(\nabla \kappa^{-1 / 3}\right)$ ) for any Killing vector field $Y \in \mathfrak{g}$. It is easy to see that the action of $G$ extends to $M$ and the dimension of the isometry group $\operatorname{Iso}(M, g)$ of $(M, g)$ is at least 4 .

$(\mathrm{c}) \Rightarrow(\mathrm{a})$. This is trivial, from $(*)$ it follows that the group $G$ of isometries of $P_{0}$ acts as isometries on $M$ with orbits $P_{0}$.

(a) $\Rightarrow(\mathrm{b})$. Since $W^{+} \neq 0$ it follows that every isometry of $M$ is holomorphic. Consequently, $X \alpha=0$ if $X \in \mathfrak{i s o}(M)$. Analogously $X\left|W^{+}\right|^{2}=0$, thus $X \kappa=0$ if $X \in \mathfrak{i s o}(M)$. Since $\theta=-\frac{2}{3} d \ln |\kappa|$ it follows that $\nabla \alpha \| \theta^{\sharp}$. Thus from Lemma $F$ we get (b).

As a corollary from the above theorem we get 
Theorem 2. Let $(M, g)$ be an oriented Einstein 4-manifold. Assume that $(M, g)$ is not locally symmetric. Then the following conditions are equivalent:

(a) $(M, g)$ is (locally) of cohomogeneity 1 (at least on an open dense subset of $M)$, with the group $\operatorname{Iso}(M)$ of (local) isometries of dimension at least 4.

(b) $(M, g)$ admits (up to change of orientation and up to two-fold covering) a compatible non-Kähler Hermitian structure $J$ such that $\left|W^{+}\right| \neq 0$ and the natural opposite almost Hermitian structure $\bar{J}$ is Hermitian.

(c) $(M, g)$ admits (up to change of orientation and up to two-fold covering) a compatible non-Kähler Hermitian structure $J$ such that the natural opposite almost Hermitian structure $\bar{J}$ is Hermitian.

(d) An open dense subset $\left(U, g_{\mid U}\right) \subset(M, g)$ is locally isometric to the manifold $\widetilde{M}=\mathbb{R} \times P_{0}$, where $\left(P_{0}, g_{0}\right)$ is a 3-dimensional $\mathcal{A}$-manifold (the total space of a Riemannian submersion $p: P_{0} \rightarrow M_{0}$ over a Riemannian surface $M_{0}$ of constant sectional curvature $\left.K \in\{-1,0,1\}\right)$ with a metric

$$
g=d t^{2}+a(t)^{2} \theta^{2}+b(t)^{2} p^{*} g_{\text {can }},
$$

where $g_{0}=\theta^{2}+p^{*} g_{\text {can }}, \theta$ is the connection form of $P_{0}$ such that $p^{*} d \theta=$ $c \operatorname{vol}_{\text {can }}, c \in \mathbb{R}$ and $\mathrm{vol}_{\text {can }}$ is the volume form of the canonical metric on $M_{0}$.

Proof. (a) $\Rightarrow$ (b). From [De-1, Lemma 9] it follows that $(M, g)$ has both tensors $W^{+}, W^{-}$degenerate. Since $(M, g)$ is not locally symmetric it follows from Proposition 1 that at least one of the functions $\left|W^{+}\right|,\left|W^{-}\right|$is not constant on $M$. Choose an orientation such that $\left|W^{+}\right|$is not constant, in particular does not vanish on $M$. Then $(M, g)$ admits a positive Hermitian non-Kähler structure $J$ (which corresponds to a simple eigenvalue of $W^{+}$) and from Theorem 1 it follows that it also admits an opposite natural Hermitian structure $\bar{J}$. Note that $\bar{J}$ is defined on an open and dense subset $U \subset M$. If $\left|W^{-}\right| \neq 0$ then $\bar{J}$ extends to the whole of $M$ as a simple eigenvalue of $W^{-}$and if $\left|W^{-}\right|$is a non-zero constant then $\bar{J}$ is Kähler.

(b) $\Rightarrow(\mathrm{c}),(\mathrm{c}) \Rightarrow(\mathrm{d})$ and $(\mathrm{d}) \Rightarrow(\mathrm{a})$ are now trivial, where we take $U=\{x \in$ $\left.M:\left|\nabla J_{x}\right| \neq 0\right\}$. Note that the metric (*) always has at least 4-dimensional group of isometries and if it is not locally symmetric then it is of cohomogeneity 1 . Note also that if $(M, g)$ admits a compatible non-Kähler Hermitian structure $J$ such that the natural opposite almost Hermitian structure $\bar{J}$ is Hermitian and $W^{+}=0$ then $W^{-} \neq 0$ and $\bar{J}$ extends to a global non-Kähler Hermitian structure such that the natural opposite structure for $\bar{J}$ is the Hermitian structure $J$ (see Lemma G), so (b) is equivalent to (c).

REMARK. Note that there are many examples of non-compact EinsteinHermitian manifolds with Hermitian natural opposite structure $\bar{J}$. For example, all the examples of A. Derdziński (see [De-2]) of self-dual Einstein- 
Hermitian structures on $\mathbb{C}^{2}$ have globally defined and Hermitian natural opposite almost Hermitian structure $\bar{J}\left(\bar{J}\right.$ is given by $\bar{J} e_{1}=-e_{3}, \bar{J} e_{2}=e_{4}$ in Derdziński's notation, clearly $\left.\mathcal{D}=\operatorname{span}\left\{e_{1}, e_{3}\right\}\right)$. Note that for arbitrary functions $a, b$ the metric $(*)$ has two Hermitian opposite structures given by the foliation $\mathcal{D}=\operatorname{span}\left\{\partial / \partial t, \theta^{\sharp}\right\}$ and distribution $\mathcal{D}^{\perp}$. The foliation $\mathcal{D}$ is totally geodesic and is contained in the nullity of $J$ and $\bar{J}$. From our theorems it follows that all such examples are generally of the form $\mathbb{R} \times P$ where $P$ is a 3 -dimensional naturally reductive manifold (the total space of a Riemannian submersion $p: P \rightarrow M_{0}$ over a Riemannian surface $\left(M_{0}\right.$, can) of constant curvature $K \in\{-1,0,1\}$ ) with the metric $g=d t^{2}+a(t)^{2} \eta \otimes \eta+b(t)^{2} p^{*}$ can where the functions $a, b$ satisfy a system of ODE's obtained by means of the O'Neill formulas so that the Einstein condition is satisfied.

\section{References}

[A-D-M] V. Apostolov, J. Davidov and O. Muškarov, Compact self-dual Hermitian surfaces, Trans. Amer. Math. Soc. 348 (1996), 3051-3063.

[A-G-1] V. Apostolov and P. Gauduchon, The Riemannian Goldberg-Sachs theorem, Internat. J. Math. 8 (1997), 421-439.

[A-G-2] —, 一, Self-dual Einstein-Hermitian four manifolds, arXiv:math.DG/0003162v3 (2000).

[B] L. Bérard-Bergery, Sur de nouvelles variétés riemanniennes d'Einstein, Inst. Élie Cartan 6, Univ. Nancy, 1982, 1-60.

[C-S-V] J. T. Cho, K. Sekigawa and L. Vanhecke, Volume-preserving geodesic symmetries on four-dimensional Hermitian Einstein manifolds, Nagoya Math. J. 146 (1997), 13-29.

[De-1] A. Derdziński, Self-dual Kähler manifolds and Einstein manifolds of dimension four, Compositio Math. 49 (1983), 405-433.

[De-2] - Exemples de métriques de Kähler et d'Einstein auto-duales sur le plan complexe, in: Géométrie Riemannienne en dimension 4, Séminaire Arthur Besse 1978-1979, Cedic Fernand Nathan, Paris, 1981, 334-346.

[D-V] F. J. E. Dillen and L. C. A. Verstraelen (eds.), Handbook of Differential Geometry, vol. I, Elsevier, 2000.

[D] T. C. Drăghici, Almost Kähler 4-manifolds with J-invariant Ricci tensor, Houston J. Math. 25 (1999), 133-145.

[G-M] G. Grantcharov and O. Muškarov, Hermitian *-Einstein surfaces, Proc. Amer. Math. Soc. 120 (1994), 233-239.

[G] A. Gray, Einstein-like manifolds which are not Einstein, Geom. Dedicata 7 (1978), 259-280.

[G-H] A. Gray and L. M. Harvella, The sixteen classes of almost Hermitian manifolds, Ann. Mat. Pura Appl. 123 (1980), 35-58.

[H] N. J. Hitchin, Kählerian twistor spaces, Proc. London Math. Soc. 43 (1981), 133-150.

[J] W. Jelonek, On A-tensors in Riemannian geometry, preprint 551, Inst. Math., Polish Acad. Sci., 1995. 
$[\mathrm{K}] \quad \mathrm{T}$. Koda, A remark on the manifold $\mathbb{C} P^{2} \sharp \overline{\mathbb{C} P^{2}}$ with Bérard-Bergery's metric, Ann. Global Anal. Geom. 11 (1993), 323-329.

[LeB] C. LeBrun, Einstein metrics on complex surfaces, in: Geometry and Physics (Aarhus, 1995), J. Andersen et al. (eds.), Lecture Notes in Pure Appl. Math. 184, Dekker, 1997, 167-176.

[M] P. Molino, Riemannian Foliations, Birkhäuser, Boston, 1988.

[P] D. Page, A compact rotating gravitational instanton, Phys. Lett. Ser. B 79 (1978), 235-238.

[P-T] H. Pedersen and P. Tod, The Ledger curvature conditions and D'Atri geometry, Differential Geom. Appl. 11 (1999), 155-162.

[P-P] J. Plebański and M. Przanowski, Hermite-Einstein four-dimensional manifolds with symmetry, Class. Quantum Grav. 15 (1998), 1721-1735.

[S-V] K. Sekigawa and L. Vanhecke, Volume-preserving geodesic symmetries on fourdimensional 2-Stein spaces, Kodai Math. J. 9 (1986), 215-224.

Institute of Mathematics

Technical University of Cracow

Warszawska 24

31-155 Kraków, Poland

E-mail: wjelon@usk.pk.edu.pl
Institute of Mathematics Polish Academy of Sciences

Cracow Branch

Św. Tomasza 30

31-027 Kraków, Poland

Reçu par la Rédaction le 30.6.2001

Révisé le 8.10.2001 et 15.4.2002 